\title{
Should we use more PET-CT in clinical cardiology?
}

\section{J. Knuuti}

Published online: 4 March 2008

(C) Springer-Verlag 2008

Recently, positron emission tomography (PET) has been increasingly used also in clinical cardiac imaging. The newest development, the hybrid imaging devices with 64slice computed tomography (CT), have become available aiming specifically to cardiac imaging and has made this technique very attractive alternative to single photon emission computed tomography (SPECT) imaging.

There are several advantages of PET as compared to SPECT. The perfusion imaging protocols with PET are faster due to shorter half-lives of the tracers and allow more efficient patient throughput. The radiation dose for the patients is only fraction of the doses provided by ${ }^{99 \mathrm{~m}} \mathrm{Tc}$ and ${ }^{201} \mathrm{Tl}$, at least when ${ }^{15} \mathrm{O}$-water or ${ }^{13} \mathrm{NH}_{3}$ are used. The image artifacts are less common, the attenuation correction is well established and less susceptible to technical problems. These technical advantages have shown to increase the specificity and accuracy in detection of coronary artery disease (CAD) [1-4]. The higher spatial resolution and heart-to-background ratios seem to lead improved detection of small perfusion defects and, thus, to higher sensitivity. Currently, PET imaging has been primarily suggested to be used in difficult patient populations such as obese and diabetic subjects.

The unique characteristic of PET is that it allows noninvasive quantification of myocardial blood flow (MBF) and metabolism. Measurement of MBF and coronary-flow reserve provides information on both the macro- and the micro-circulation and likely more accurate detection of both early and advanced disease [5-7].

This Editorial commentary refers to the article http://dx.doi.org/ 10.1007/s00259-007-0647-3.

J. Knuuti $(\bowtie)$

Turku PET Centre, University of Turku,

P.O. Box 52, 20521 Turku, Finland

e-mail: juhani.knuuti@utu.fi
Do these technical advantages and improved accuracy justify the use of PET in clinical routine? Myocardial perfusion SPECT has a huge amount of evidence supporting its robustness and accuracy in the detection of CAD, and its prognostic value is proven. The strengths and limitations of the technique are well known. Experienced staff is existing to deliver high quality service worldwide. It is obvious that to overtake the role of SPECT perfusion imaging is not an easy task for any technique.

The limitations of PET are also currently obvious. While the availability of scanners is rapidly improving, the tracer production remains a major bottleneck. Without large multi-centre trials documenting the improved accuracy leading to improved patient care or cost savings, the justification of wider use of PET is difficult. Subsequently, without wider utilisation of the technique, new investments to PET tracer production are not sustainable.

In the current issue of this journal, Siegrist et al. [8] investigated the impact of ${ }^{13} \mathrm{~N}$-ammonia myocardial PET perfusion imaging on clinical decision-making and the costeffectiveness in 100 consecutive patients with high likelihood of CAD or known CAD. The patient management decisions were assessed without and with the knowledge of PET results. The authors found that the management changed in 78 patients of 100 using PET perfusion information. In addition, the final clinical management decisions were in agreement with the PET findings in $97 \%$ of the cases. Cost-effectiveness analysis revealed significant costs savings as a result of using PET.

The study is interesting and provides us clinically useful information. It demonstrates that in the centre with established cardiac PET service, clinicians trust on the results and plan their treatment strategies based the findings. The limitations of the study are related to the fact that the study was not prospectively planned only for this purpose and, therefore, not all clinical end-points such as long-term 
clinical patient follow-up were available. The classification of the management strategies in the study leads to some overestimation of the clinical impact due to fact that diagnostic angiography and diagnostic angiography and PCI represented two different treatment strategies in the study, although in real life, there is tight linkage between these strategies, typically the first often leading to the second.

On the other hand, this phenomenon likely leads underestimation of the true savings because if the patients had been referred for diagnostic angiography without PET perfusion imaging, number of subsequent angioplasties following coronary angiography would have been higher and the costs increased further. However, this kind of analysis is based on several uncertain assumptions and was ignored in the paper. The same is also true with the potential changes in the medical therapy costs.

In the present study by Siegrist et al. [8], CT was used only for attenuation correction. In future, it would be very interesting to study the clinical impact and cost-effectiveness of true hybrid imaging with $\mathrm{CT}$ angiography and perfusion imaging in a real clinical setting. Furthermore, the pre-test likelihood of CAD was very high in the present study while non-invasive imaging is likely at its best in a population with moderate pre-test likelihood. Furthermore, the unique characteristic of PET, the possibility to measure myocardial blood flow in quantitative terms was not included in the analysis [8].

Finally, although current focus is still in the detection of CAD, it should be kept in mind that the greatest potential of PET in clinical cardiology is likely in the field of molecular imaging. The future targets will be such as imaging of inflammation, vulnerable plaques and monitoring of gene and stem cell therapies.

\section{References}

1. Klocke FJ, Baird MG, Lorell BH, et al. ACC/AHA/ASNC guidelines for the clinical use of cardiac radionuclide imagingexecutive summary: a report of the American College of Cardiology/American Heart Association Task Force on Practice Guidelines (ACC/AHA/ASNC Committee to Revise the 1995 Guidelines for the Clinical Use of Cardiac Radionuclide Imaging). Circulation 2003;108:1404-18.

2. Marwick TH, Shan K, Patel S, Go RT, Lauer MS. Incremental value of rubidium- 82 positron emission tomography for prognostic assessment of known or suspected coronary artery disease. Am J Cardiol. 1997;80:865-70.

3. Di Carli MF, Hachamovitch R. New technology for noninvasive evaluation of coronary artery disease. Circulation 2007;115 (11):1464-80. Mar 20.

4. Muzik O, Duvernoy C, Beanlands RS, Sawada S, Dayanikli F, Wolfe ER Jr, et al. Assessment of diagnostic performance of quantitative flow measurements in normal subjects and patients with angiographically documented coronary artery disease by means of nitrogen-13 ammonia and positron emission tomography. J Am Coll Cardiol. 1998;31(3):534-40. Mar 1.

5. Yoshinaga K, Katoh C, Noriyasu K, Iwado Y, Furuyama H, Ito Y, et al. Reduction of coronary flow reserve in areas with and without ischemia on stress perfusion imaging in patients with coronary artery disease: a study using oxygen 15-labeled water PET. J Nucl Cardiol. 2003;10(3):275-83. May-Jun.

6. Parkash R, deKemp RA, Ruddy TD, Kitsikis A, Hart R, Beauchesne L, et al. Potential utility of rubidium 82 PET quantification in patients with 3-vessel coronary artery disease. J Nucl Cardiol. 2004;11(4):440-9. Jul-Aug.

7. Santana CA, Folks RD, Garcia EV, Verdes L, Sanyal R, Hainer J, et al. Quantitative (82)Rb PET-CT: development and validation of myocardial perfusion database. J Nucl Med. 2007;48(7):1122-8. Jul.

8. Siegrist PT, Husmann L, Knabenhans M, Gaemperli O, Valenta I, Hoefflinghaus T, Scheffel H, Stolzmann P, Alkadhi H, Kaufmann PA. 13N-Ammonia Myocardial Perfusion Imaging with PET-CT scanner: Impact on Clinical Decision-Making and Cost-Effectiveness. Eur J Nucl Med Mol Imaging 2007(in press). 